\title{
NOTE ON KADETS KLEE PROPERTY AND ASPLUND SPACES
}

\author{
PETR HÁJEK AND JARNO TALPONEN \\ (Communicated by Thomas Schlumprecht)
}

\begin{abstract}
A typical result in this note is that if $X$ is a Banach space which is a weak Asplund space and has the $\omega^{*}-\omega$-Kadets Klee property, then $X$ is already an Asplund space.
\end{abstract}

\section{INTRODUCTION}

Recall that a Banach space $(X,\|\cdot\|)$ (resp. its norm $\|\cdot\|$ ) is said to be locally uniformly rotund (LUR for short) if for each $x, x_{n} \in X, n \in \mathbb{N}$, with $\|x\|=\left\|x_{n}\right\|=1$ and $\lim _{n \rightarrow \infty}\left\|x_{n}+x\right\|=2$ it follows that $\lim _{n \rightarrow \infty}\left\|x_{n}-x\right\|=0$.

We say that a Banach space $(X,\|\cdot\|)$ has the Kadec property (resp. Kadec-Klee property, KK for short) if the norm and weak topologies coincide on the unit sphere $S_{X}$ (resp. weakly convergent sequences from $S_{X}$ are norm convergent).

In [Tro3] it is shown that if $\ell_{1} \leftrightarrow X$ and $\|\cdot\|$ is KK, then it is also Kadec. The Kadec property has been studied extensively in the literature, and we refer to [DGZ and [MOTV] for the background. Let us mention only a few of the main results in this area which relate the Kadec and LUR properties. It is easy to observe that if $\|\cdot\|$ is LUR, then $\|\cdot\|$ has the Kadec property. Troyanski proved [Tro1], Tro2 that $X$ has equivalent LUR renorming if and only if it has equivalent Kadec and rotund renormings. Haydon Hay, Hayd3 gave examples showing that the rotundity assumption is essential, constructing trees such that $C(T)$ has KK but no rotund renorming. In [MOTV] the authors prove that if $X$ has the RNP property (in particular if $X$ is a dual of some Asplund space), then $X$ has an LUR renorming if and only if it has a Kadec renorming. In the present note we are interested in the dual situation.

Definition 1.1. Let $(X,\|\cdot\|)$ be a Banach space. We say that $\left(X^{*},\|\cdot\|^{*}\right)$ has the

1. Dual Kadec property $\left(\mathrm{K}^{*}\right)$ if the $w^{*}$ and norm topologies coincide on the dual unit sphere $S_{X^{*}}$.

Received by the editors August 21, 2012 and, in revised form, December 11, 2012 and December 19, 2012.

2010 Mathematics Subject Classification. Primary 46B03, 46B20.

Key words and phrases. Asplund spaces, weak-star Kadets Klee property, weak-star-to-weak Kadets Klee property, Grothendieck spaces, renormings, duality mapping, WLD, weakly Lindelöf determined, SCP, separable complementation property, coseparable subspaces.

The first author was financially supported by grant GACR 201/11/0345 and was supported in part by Institutional Research Plan AV0Z10190503 and GAČR P201/11/0345.

This paper was prepared as the second author enjoyed the warm hospitality of the Czech Academy of Sciences in autumn 2011. The visit and research were supported in part by the Väisälä Foundation. 
2. Dual weak Kadec property $\left(\mathrm{wK}^{*}\right)$ if the $w^{*}$ and weak topologies coincide on the dual unit sphere $S_{X^{*}}$.

3. Dual Kadec-Klee property $\left(\mathrm{KK}^{*}\right)$ if the $w^{*}$-convergent and norm convergent sequences coincide on the dual unit sphere $S_{X^{*}}$.

4. Dual weak Kadec-Klee property $\left(\mathrm{wKK}^{*}\right)$ if the $w^{*}$-convergent and weak convergent sequences coincide on the dual unit sphere $S_{X^{*}}$.

These notions are closely related to the Asplund property of $X$. In particular, Namioka and Phelps [NP showed that if $X^{*}$ has the $\mathrm{K}^{*}$, then $X$ is an Asplund space. Raja strengthened this by showing that $X^{*}$ has a dual LUR renorming (which implies automatically the $\mathrm{K}^{*}$ property and also that $X$ is an Asplund space) if and only if it has a $\mathrm{K}^{*}$ renorming if and only if it has a $\mathrm{wK}^{*}$ renorming [R1, $\mathrm{R} 2$.

It is therefore natural to ask whether the formally weaker sequential versions of these properties lead to some positive result. The problem of whether the existence of a $\mathrm{KK}^{*}$ renorming of $X^{*}$ implies that $X$ is an Asplund space has apparently been posed by Godefroy, and it seems to be open. In our note we are going to address the still weaker property $\mathrm{wKK}^{*}$ of $X^{*}$. It follows that for every renorming of a given Grothendieck space $X$, its dual $X^{*}$ has the wKK* . Hence the property may not imply that $X$ is Asplund, in general (e.g., $\ell_{\infty}$ ).

1.1. Preliminaries. We denote by $X, Y, Z$ and $E$ real Banach spaces. The closed unit ball and the unit sphere of $X$ are denoted by $B_{X}$ and $S_{X}$, respectively. For suitable background information on general Banach space theory and notations we refer to [FHHMZ].

We say that a subspace $Y \subset X$ is coseparable in $X$ if $X / Y$ is separable (see [T] for discussion). Following [F] we call $X$ a Gateaux differentiability space (GDS) if each continuous convex function on $X$ is Gateaux differentiable in a dense set. If the sets in the previous definition are additionally $G_{\delta}$-sets, then $X$ is a weak Asplund space. A Banach space $X$ has the Grothendieck property if in the dual $X^{*}$ the $w^{*}$-convergent and $w$-convergent sequences coincide. Recall that a Banach space has the 1-separable complementation property (1-SCP) if for each separable subspace $Y \subset X$ there exists a separable subspace $Z \subset X$ such that $Y \subset Z$ and $Z$ is contractively complemented in $X$. If $A \subset X$ is a subset, then we denote by $[A]$ the closed linear span of $A$. The duality mapping $J: \mathbf{S}_{X} \rightarrow 2^{\mathbf{S}_{X^{*}}}$ is given by $J(x)=\left\{x^{*} \in \mathbf{S}_{X^{*}}: x^{*}(x)=1\right\}$ for $x \in \mathbf{S}_{X}$.

\section{Results}

Theorem 2.1. Let $X$ be a Banach space which can be renormed in such a way that $X^{*}$ satisfies both the $w K K^{*}$ and one of the following conditions:

(i) $J: \mathbf{S}_{X} \rightarrow 2^{\mathbf{S}_{X}^{*}}$ is $w^{*}$-first-countably valued.

(ii) The coseparable subspaces of $X$ are preserved in countable intersections.

(iii) $X$ has 1-SCP.

(iv) $\mathbf{B}_{X^{*}}$ is $w^{*}$-countably compact (e.g., $X$ is a Gateaux differentiability space or weak Asplund).

Then $X$ is an Asplund space. 
Observe that the wKK* property of $X^{*}$ is clearly weaker than the $\mathrm{KK}^{*}$ property and the Grothendieck property. Since there exists non-Asplund Grothendieck spaces, e.g., $L^{\infty}$, we conclude that the assumptions $(i)-(i v)$ cannot be plainly removed in the theorem. Shortly we will discuss the conditions $(i)$ and $(i i)$. The first condition is of course much weaker than imposing $J$ to be single-valued, i.e., $X$ to be Gateaux smooth. Recall that WLD, Plichko and certain other classes of Banach spaces have 1-SCP; see [HMVZ, p. 105].

Proposition 2.2. Let $x^{*} \in \mathbf{S}_{X^{*}}, x \in \mathbf{S}_{X}$ be such that $x^{*}(x)=1$. Let $\left(x_{n}^{*}\right) \subset \mathbf{B}_{X^{*}}$ be a sequence such that $x^{*} \in{\overline{\left(x_{n}^{*}\right)}}^{w^{*}}$. Assume that there exists a sequence $\left(U_{n}\right)$ of relatively $w^{*}$-open subsets of $J(x)$ such that

$$
\bigcup_{n} U_{n}=\bigcup_{n}{\overline{U_{n}}}^{w^{*}}=J(x) \backslash\left\{x^{*}\right\} .
$$

Then there exists a subsequence $\left(n_{k}\right) \subset \mathbb{N}$ such that $x_{n_{k}}^{*} \stackrel{w^{*}}{\longrightarrow} x^{*}$ as $k \rightarrow \infty$.

First we will comment on the assumptions regarding $J(x)$ in the $w^{*}$-topology, which is subsequently termed $K$. If $J(x)$ is norm-separable, then it is, in particular, hereditarily Lindelöf in the $w^{*}$-topology. The same is true if $K$ is metrizable. Recall that a compact hereditarily Lindelöf space is first-countable (see e.g. [BG, p. 473]).

The following condition is also useful: There exists a sequence $\left(x_{n}\right) \subset X \subset X^{* *}$ such that the sequence $y_{n}=\left.x_{n}\right|_{[J(x)]} \in[J(x)]^{*}$ satisfies

$$
\overline{\left[\left(y_{n}\right)\right]} w^{*}=[J(x)]^{*} ;
$$

that is, the sequence $\left(x_{n}\right)$ separates $[J(x)]$. This condition, or the first-countability of $K$, implies, in particular, that there exists a sequence $\left(x_{n}\right) \subset X$ such that for any $y^{*} \in K, y^{*} \neq x^{*}$, there is $n$ such that $\left(y^{*}-x^{*}\right)\left(x_{n}\right) \neq 0$. Observe that this condition in turn implies the topological assumption of Proposition 2.2 .

Proof of Proposition 2.2. Fix a sequence $\left(U_{n}\right)$ of $w^{*}$-open subsets of $K$ such that

$$
\bigcup_{n} U_{i}=\bigcup_{n}{\overline{U_{i}}}^{w^{*}}=K \backslash\left\{x^{*}\right\} .
$$

Let $\left(\tilde{U}_{n}\right)$ be a sequence of $w^{*}$-open sets of $\mathbf{B}_{X^{*}}$ such that $U_{n} \subset \tilde{U}_{n} \cap J(x)$ and $x^{*} \notin{\tilde{\tilde{U}_{n}}}^{w^{*}}$ for $n \in \mathbb{N}$. Indeed, $K$ is a compact Hausdorff space, thus normal, hence completely regular, and so we may separate $\overline{U_{n}}{ }^{*}$ and $x^{*}$ by a continuous function $g: K \rightarrow[0,1]$. Then a Tietze extension $\tilde{g}: \mathbf{B}_{X^{*}} \rightarrow[0,1]$ of $g$ yields a suitable $w^{*}$ open set $\tilde{U}_{n}$. Let $\left(V_{i}\right)$ be a sequence defined by $V_{2 i}=\tilde{U}_{i}$ and $V_{2 i-1}=\left\{y^{*} \in \mathbf{B}_{X^{*}}\right.$ : $\left.x\left(y^{*}\right)<1-1 / i\right\}$.

We define a subsequence of $\left(x_{n}^{*}\right)$ by a diagonal argument as follows. First, we remove from the sequence the functionals included in $V_{1}$. From the resulting sequence, say $s_{1}$, we remove the functionals included in $V_{2}$, starting from the index third in order. Next, from the previously obtained sequence $s_{2}$ we pick a new sequence $s_{3}$ by removing the functionals included in $V_{3}$ starting from the fourth index. We proceed in this manner. Observe that $s_{n}$ includes the $n$ first elements of $s_{n-1}$. Therefore $s_{n}$ converges coordinate-wise to a sequence $s$, which is a subsequence of $\left(x_{n}^{*}\right)$. Here $\left(x_{n}\right) \supset s_{1} \supset s_{2} \supset \ldots \supset s$. Let us denote this resulting sequence $s$ by $\left(x_{n_{k}}^{*}\right)$. By the construction $x_{n_{k}}^{*}(x) \rightarrow 1$ as $k \rightarrow \infty$. 
Assume to the contrary that $x_{n_{k}}^{*}$ does not $w^{*}$-converge to $x^{*}$. Thus there is a point $y \in X$ and a further subsequence $\left(n_{k_{j}}\right)_{j}$ such that $\left(x^{*}-x_{n_{k_{j}}}^{*}\right)(y) \rightarrow c \neq 0$ as $j \rightarrow \infty$. By the $w^{*}$-compactness of $\mathbf{B}_{X^{*}}$ we get that there is $z^{*} \in J(x), z^{*} \neq x^{*}$, such that $z^{*}$ is a $w^{*}$-cluster point of $\left(x_{n_{k_{j}}}^{*}\right)_{j}$. Then, according to the assumptions there is $i_{0} \in \mathbb{N}$ such that $z^{*} \in \tilde{U}_{i_{0}}$. By reviewing the construction of $s_{2 i_{0}}$ we observe the impossibility of $z^{*}$ being a $w^{*}$-cluster point of $s_{2 i_{0}}$. This contradiction finishes the proof.

Proposition 2.3. Let $X$ be a Banach space which can be renormed to be both GDS and 1-SCP. Then the coseparable subspaces of $X$ are preserved in taking countable intersections.

Proof. Let $X$ satisfy the assumption about the renorming. Since the conclusion of the result obviously does not depend on equivalent renormings, we may restrict our attention to a given 1-SCP Gateaux differentiability space $X$. Let $\left(Z_{n}\right)$ be a sequence of coseparable subspaces of $X$. We denote by $q_{n}: X \rightarrow X / Z_{n}$ the canonical quotient mappings.

By applying the fact that $X / Z_{n}$ are separable and the GDS property we may choose subsets $\left\{z_{k}^{(n)} \in \mathbf{S}_{X}: n, k \in \mathbb{N}\right\}$ and $\left\{f_{k}^{(n)} \in \mathbf{S}_{X^{*}}: n, k \in \mathbb{N}\right\}$ such that:

(a) Each $z_{k}^{(n)}$ is a smooth point.

(b) $\mathbf{S}_{X / Z_{n}} \subset q_{n}\left(\left\{z_{k}^{(n)}: k \in \mathbb{N}\right\}\right)$ for each $n$.

(c) Each $f_{k}^{(n)}$ attains its norm at $z_{k}^{(n)}$.

(d) $\left\{f_{k}^{(n)}: k \in \mathbb{N}\right\}$ 1-norms $\left[z_{k}^{(n)}: k \in \mathbb{N}\right]$ for each $n$.

Indeed, the last condition is obtained by applying a back-and-forth recursion of countable length. From (b) and (d) we obtain that $\mathbf{B}_{Z_{n}^{\perp}} \subset \overline{\operatorname{conv}} w^{*}\left(f_{k}^{(n)}: k \in \mathbb{N}\right)$ for $n$. This means that

$$
\bigcap_{k} \operatorname{ker} f_{k}^{(n)} \subset Z_{n} \quad \text { for } n \in \mathbb{N} \text {. }
$$

According to 1-SCP there exists a separable subspace $E \subset X$ with $\left\{z_{k}^{(n)}: n, k \in\right.$ $\mathbb{N}\} \subset E$ and a norm-1 linear projection $P: X \rightarrow E$.

According to the Gateaux smoothness of the points $z_{k}^{(n)}$ we have that

$$
f_{k}^{(n)} \circ P=f_{k}^{(n)} \quad \text { for } n, k \in \mathbb{N},
$$

since the functionals are norm attaining at the corresponding points. Thus ker $P \subset$ $\bigcap_{n, k} \operatorname{ker} f_{k}^{(n)}$ where ker $P$ is clearly a coseparable subspace. On the other hand, $\bigcap_{k} \operatorname{ker} f_{k}^{(n)} \subset Z_{n}$ for $n$, so that $\bigcap_{n} Z_{n}$ is necessarily a coseparable subspace of $X$.

Proof of Theorem 2.1. Let $Y \subset X$ be a separable subspace. Our aim is to show that $Y^{*}$ is separable.

Observe that $\mathbf{B}_{Y^{*}}$ is separable and metrizable in the $w^{*}$-topology. It is easy to see that $\mathbf{S}_{Y^{*}}$ is such as well. Let us pick a $w^{*}$-dense set $\left\{y_{n}^{*}: n \in \mathbb{N}\right\} \subset \mathbf{S}_{Y^{*}}$. Our aim is to show that

$$
\overline{\operatorname{conv}}\left(\left\{y_{n}^{*}: n \in \mathbb{N}\right\}\right)=\mathbf{B}_{Y^{*}} .
$$

Towards this, fix a norm-attaining functional $y^{*} \in \mathbf{S}_{Y^{*}}$. Let $y \in \mathbf{S}_{Y}$ be such that $y^{*}(y)=1$. 
By using $w^{*}$-compactness and $w^{*}$-metrizability of the dual unit ball we obtain that there exists a subsequence $\left(y_{n_{k}}^{*}\right)_{k \in \mathbb{N}} \subset\left\{y_{n}^{*}: n \in \mathbb{N}\right\}$ which $w^{*}$-converges to $y^{*}$, since the countable set was chosen to be $w^{*}$-dense.

Our aim is to find a further subsequence of $\left(n_{k}\right)$, indexed by $m$, and a sequence $\left(z_{m}^{*}\right)_{m \in \mathbb{N}} \subset \mathbf{S}_{X^{*}}$ and $z^{*} \in \mathbf{S}_{X^{*}}$, which are Hahn-Banach extensions of $\left(y_{n_{k_{m}}}^{*}\right)_{m \in \mathbb{N}}$ and $y^{*}$, respectively, such that $z_{m}^{*} \stackrel{w^{*}}{\longrightarrow} z^{*}$ in $X^{*}$ as $m \rightarrow \infty$. Namely, in such a case we obtain by the assumptions that $z_{m}^{*} \stackrel{w}{\longrightarrow} z^{*}$ and then Mazur's theorem yields that $z^{*} \in \overline{\operatorname{conv}}\left(z_{m}^{*}: m \in \mathbb{N}\right)$. This means that $y^{*} \in \overline{\operatorname{conv}}\left(y_{n_{k_{m}}}^{*}: m \in \mathbb{N}\right)$, and since $y^{*}$ was an arbitrary norm-attaining functional, we obtain by the Bishop-Phelps theorem that $\overline{\operatorname{conv}}\left(y_{n}^{*}: n \in \mathbb{N}\right)=\mathbf{B}_{Y^{*}}$.

Fix Hahn-Banach extensions $\left(x_{k}^{*}\right)$ and $x^{*}=x_{0}^{*}$ of $\left(y_{n_{k}}^{*}\right)$ and $y^{*}$, respectively. Observe that $x_{k}^{*}(y) \rightarrow 1=x_{0}^{*}(y)$ as $k \rightarrow \infty$. To finish the proof by finding the appropriate subsequence $\left(n_{k_{m}}\right)_{m}$ and Hahn-Banach extensions, we proceed in parallel steps regarding assumptions (i)-(iv).

Assumption (i): The set $J(y)$ is $w^{*}$-first-countable. Proposition 2.2 and the discussion following yield that there is a subsequence $\left(k_{m}\right)$ and $z^{*} \in J(y) \subset \mathbf{S}_{X^{*}}$ such that $x_{k_{m}}^{*} \stackrel{w^{*}}{\longrightarrow} z^{*}$ as $m \rightarrow \infty$. Note that $z^{*}$ is a Hahn-Banach extension of $y^{*}$, since by the selection of $\left(x_{k_{m}}^{*}\right)$ it holds that $\left.x_{k_{m}}^{*}\right|_{Y} \stackrel{w^{*}}{\longrightarrow} y^{*}$ in $Y^{*}$ as $m \rightarrow \infty$. We write $z_{m}^{*}=x_{k_{m}}^{*}$ to get the required extensions.

Assumption (ii): The coseparable subspaces of $X$ are preserved in countable intersections. Observe that $Z=\bigcap_{k>0} \operatorname{ker} x_{k}^{*} \subset X$ is coseparable. Thus we may consider $\left\{x_{k}^{*}: k \geq 0\right\} \subset \mathbf{B}_{(X / Z)^{*}}=\mathbf{B}_{Z^{\perp}}$, where the unit ball is $w^{*}$-compact and $w^{*}$-metrizable. Thus we may extract a $w^{*}$-converging subsequence $\left(x_{k_{m}}^{*}\right)$. Let $z^{*}=w^{*}-\lim _{m \rightarrow \infty} x_{k_{m}}^{*}$. We put $z_{m}^{*}=x_{k_{m}}^{*}$ for $m$. By the selection of the sequence $\left(n_{k_{m}}\right)$ it is again clear that $z^{*} \in \mathbf{S}_{X^{*}}$ is a Hahn-Banach extension of $y^{*} \in \mathbf{S}_{Y^{*}}$.

Assumption (iii): $X$ has 1-SCP. Let $P: X \rightarrow E$ be a norm-1 projection where $E \subset X$ is a separable subspace containing $Y$. As in the previous step, we observe that $\left\{x_{k}^{*} \circ P: k \geq 0\right\} \subset \mathbf{B}_{(X / \operatorname{ker} P)^{*}}$ contains a subsequence $\left(z_{m}^{*}\right)=\left(x_{k_{m}}^{*} \circ P\right)$ and $z^{*}=z^{*} \circ P \in \mathbf{S}_{(X / \operatorname{ker} P)^{*}}, z^{*}(y)=1$, such that $x_{k_{m}}^{*} \circ P \stackrel{w^{*}}{\longrightarrow} z^{*}$ in $(X / \operatorname{ker} P)^{*}$ as $m \rightarrow \infty$. Since $Y \subset E$, we see similarly as above that $\left.\left(x_{k_{m}}^{*} \circ P\right)\right|_{Y}=y_{n_{k_{m}}}^{*}$ for $m \in \mathbb{N}$. This yields that $\left.z^{*}\right|_{Y}=y^{*}$.

Assumption (iv): The dual unit ball $\mathbf{B}_{X^{*}}$ is $w^{*}$-sequentially compact. This is the case, for instance, if $X$ is GDS (see [F. Theorem 2.1.2]). Thus there is a subsequence $\left(k_{m}\right) \subset \mathbb{N}$ and $z^{*} \in \mathbf{S}_{X^{*}}$ such that $z^{*}(y)=1$ and $w^{*}-\lim _{m \rightarrow \infty} x_{k_{m}}^{*}=z^{*}$. Clearly $\left.z^{*}\right|_{Y}=y^{*}$ and we put $z_{m}^{*}=x_{k_{m}}^{*}$.

Finally, we will apply Theorem 2.1 to give a new, rather simple proof for the Josefson-Nissenzweig Theorem in a subclass of Banach spaces.

Proposition 2.4. Let $X$ be an infinite-dimensional Banach space which is not an Asplund space and satisfies one of the conditions (i)-(iv) in Theorem 2.1. Then there is a sequence $\left(z_{k}^{*}\right) \subset \mathbf{S}_{X^{*}}$ such that $w^{*}-\lim _{k \rightarrow \infty} z_{k}^{*}=0$ and $0 \notin \overline{\operatorname{conv}}\left(z_{k}^{*}\right)$.

Proof. Let $Y \subset X$ be a separable subspace with non-separable dual. Thus $Y$ is not Asplund but $\mathbf{B}_{Y^{*}}$ is $w^{*}$-metrizable. By using Theorem 2.1 and its proof we conclude 
that there exist a sequence $\left(y_{n}^{*}\right) \subset \mathbf{S}_{Y^{*}}$ and a point $y^{*} \in \mathbf{S}_{Y^{*}}$ such that $y_{n}^{*} \stackrel{w^{*}}{\longrightarrow} y^{*}$ as $n \rightarrow \infty$ but $y^{*} \notin \overline{\operatorname{conv}}\left(y_{n}^{*}\right)$. By proceeding similarly as in the proof of Theorem 2.1 we may find a subsequence $\left(n_{k}\right)$ and Hahn-Banach extensions $x_{n_{k}}^{*}$ and $x^{*}$ of $y_{n_{k}}^{*}$ and $y^{*}$, respectively, such that $x_{n_{k}}^{*} \stackrel{w^{*}}{\longrightarrow} x^{*}$ in $X^{*}$ as $k \rightarrow \infty$. Define $u_{k}^{*}=x_{n_{k}}^{*}-x^{*}$ for $k$. This is a $w^{*}$-null sequence. Observe that $0 \notin \overline{\operatorname{conv}}\left(u_{k}^{*}\right)$ since $y^{*} \notin \overline{\operatorname{conv}}\left(y_{n}^{*}\right)$. By the geometric Hahn-Banach theorem there is a separating functional $f \in X^{* *}$ such that, say, $f\left(u_{k}^{*}\right)>1$ for $k$. We define the required sequence by $z_{k}^{*}=u_{k}^{*} /\left\|u_{k}^{*}\right\|$ where $\left\|u_{k}^{*}\right\| \leq 2$ for all $k \in \mathbb{N}$. Observe that $f\left(z_{k}^{*}\right)>1 / 2$ for $k \in N$, so that $0 \notin \overline{\operatorname{conv}}\left(z_{k}^{*}\right)$.

2.1. Final remarks. Regarding the above-mentioned problem of Godefroy, the example $[\mathrm{Fe}$ ] of countably tight compact $K$ without any convergent sequence provides a possible direction for searching a counterexample $C(K)$ space.

Problem 2.5. Find examples with $K K$ failing $K$, resp. wKK* failing $K^{*}$. Consider the long James space $J(\Gamma)$. Does it have the $\mathrm{K}^{*}$ ?

Another related subject is the following weakening of the well-known problem of whether every dual to a separable space without a copy of $\ell_{1}$ is LUR renormable (see e.g. [HMO]).

Problem 2.6. Let $X$ be separable $\ell_{1} \nrightarrow \rightarrow$. Does $X^{*}$ admit a KK renorming?

\section{REFERENCES}

[BG] Murray Bell and John Ginsburg, Uncountable discrete sets in extensions and metrizability, Canad. Math. Bull. 25 (1982), no. 4, 472-477, DOI 10.4153/CMB-1982-068-3. MR674565 (84c:54009)

[BoP] Piotr Borodulin-Nadzieja and Grzegorz Plebanek, On sequential properties of Banach spaces, spaces of measures and densities, Czechoslovak Math. J. 60(135) (2010), no. 2, 381-399, DOI 10.1007/s10587-010-0042-2. MR2657956 (2011g:46025)

[DGZ] Robert Deville, Gilles Godefroy, and Václav Zizler, Smoothness and renormings in Banach spaces, Pitman Monographs and Surveys in Pure and Applied Mathematics, vol. 64, Longman Scientific \& Technical, Harlow, 1993. MR1211634 (94d:46012)

[Dieu] J. Dieudonné, Foundations of modern analysis, Pure and Applied Mathematics, vol. X, Academic Press, New York, 1960. MR0120319 (22 \#11074)

[F] Marián J. Fabian, Gâteaux differentiability of convex functions and topology. Weak Asplund spaces, Canadian Mathematical Society Series of Monographs and Advanced Texts, A Wiley-Interscience Publication, John Wiley \& Sons Inc., New York, 1997. MR:1461271 (98h:46009)

[FHHMZ] Marián Fabian, Petr Habala, Petr Hájek, Vicente Montesinos, and Václav Zizler, Banach space theory. The basis for linear and nonlinear analysis, CMS Books in Mathematics/Ouvrages de Mathématiques de la SMC, Springer, New York, 2011. MR2766381(2012h:46001)

[Fe] V. V. Fedorčuk, A compact space having the cardinality of the continuum with no convergent sequences, Math. Proc. Cambridge Philos. Soc. 81 (1977), no. 2, 177-181. MR0425877 (54 \#13827)

[HMVZ] Petr Hájek, Vicente Montesinos Santalucía, Jon Vanderwerff, and Václav Zizler, Biorthogonal systems in Banach spaces, CMS Books in Mathematics/Ouvrages de Mathématiques de la SMC, 26, Springer, New York, 2008. MR2359536(2008k:46002)

[Hay] Richard Haydon, A counterexample to several questions about scattered compact spaces, Bull. London Math. Soc. 22 (1990), no. 3, 261-268, DOI 10.1112/blms/22.3.261. MR.1041141 (91h:46045)

[Hayd3] Richard Haydon, Trees in renorming theory, Proc. London Math. Soc. (3) 78 (1999), no. 3, 541-584, DOI 10.1112/S0024611599001768. MR1674838(2000d:46011) 
[HMO] R. Haydon, A. Moltó, and J. Orihuela, Spaces of functions with countably many discontinuities, Israel J. Math. 158 (2007), 19-39, DOI 10.1007/s11856-007-0002-1. MR2342456(2008k:46046)

[Me] Sophocles Mercourakis, Some remarks on countably determined measures and uniform distribution of sequences, Monatsh. Math. 121 (1996), no. 1-2, 79-111, DOI 10.1007/BF01299640. MR1375642(97j:28029)

[MOTV00] Aníbal Moltó, José Orihuela, Stanimir Troyanski, and Manuel Valdivia, Kadec and Krein-Milman properties (English, with English and French summaries), C. R. Acad. Sci. Paris Sér. I Math. 331 (2000), no. 6, 459-464, DOI 10.1016/S0764-4442(00)01644X. MR $1792486(2001 \mathrm{i}: 46018)$

[MOTV] Aníbal Moltó, José Orihuela, Stanimir Troyanski, and Manuel Valdivia, A nonlinear transfer technique for renorming, Lecture Notes in Mathematics, vol. 1951, SpringerVerlag, Berlin, 2009. MR2462399 (2010a:46018)

[NP] I. Namioka and R. R. Phelps, Banach spaces which are Asplund spaces, Duke Math. J. 42 (1975), no. 4, 735-750. MR0390721 (52 \#11544)

[R2] M. Raja, On dual locally uniformly rotund norms, Israel J. Math. 129 (2002), 77-91, DOI 10.1007/BF02773154. MR.1910933 (2003f:46027)

[R1] M. Raja, Locally uniformly rotund norms, Mathematika 46 (1999), no. 2, 343-358, DOI 10.1112/S0025579300007816. MR.1832626 (2002e:46019)

[T] Jarno Talponen, Extracting long basic sequences from systems of dispersed vectors, Adv. Math. 231 (2012), no. 2, 1068-1084, DOI 10.1016/j.aim.2012.06.008. MR.2955202

[Tro1] S. L. Trojanski, Locally uniformly convex norms (Russian), C. R. Acad. Bulgare Sci. 32 (1979), no. 9, 1167-1169. MR562928 (81b:46015)

[Tro2] S. L. Troyanski, Construction of equivalent norms for certain local characteristics with rotundity and smoothness by means of martingales. (Russian. English summary), Proc. 14th Spring Conf. Union of Bulgarian Mathematicians (1985), B"lgar. Akad. Nauk, Sofia, 1985, pp. 129-156. MR805345 (87c:46027)

[Tro3] S. L. Troyanski, On a property of the norm which is close to local uniform rotundity, Math. Ann. 271 (1985), no. 2, 305-313, DOI 10.1007/BF01455991. MR783556 (86g:46030)

Mathematical Institute, Czech Academy of Science, Žitná 25, 11567 Praha 1, Czech Republic - And - Department of Mathematics, Faculty of Electrical Engineering, Czech Technical University in Prague, Zikova 4, 160 00, Prague, Czech Republic

E-mail address: hajek@math.cas.cz

Aalto University, Institute of Mathematics, P.O. Box 11100, Fi-00076 Aalto, Finland Current address: University of Eastern Finland, Institute of Mathematics, Box 111, FI-80101 Joensuu, Finland

E-mail address: talponen@iki.fi 